\title{
WHEN DO WE NEED PLEURAL SURGERY?*
}

Paul L Tahalele ${ }^{1)}$, Merlinda Dwintasar ${ }^{1)}$, Y Motulo $^{2)}$, Yan Efrata Sembiring ${ }^{3)}$, Dhihintia Jiwangga Suta ${ }^{3)}$

\section{ABSTRACT}

Pleural sac is an enclosed sac covering the lung that consist of two membranes, i.e. parietal and visceral pleura. It has a cavity filled with small amount fluid that plays important role to provide smooth sliding of lung during respiration. Change or disturbance in the pleural cavity and or the pleura itself may result in restriction of lung expansion causing ventilation compromise.

Pathology of pleura may result from varying etiology. It may present as inflammatory pleural effusion, non-inflammatory pleural effusion, pneumothorax, hematothorax, fibrothorax, pleural tumors, and cylothorax. Diagnostic tool for pleural diseases may be made from the simplest chest $x$-ray, ultrasound, computed tomography, MRI and histopathological studies.

There are several treatment options for managing pleural diseases. It consist non operatif treatment, pleural drainage, decortication or pleurectomy, pleurodesis, and/ or resection of pleural tumor through procedure VATS, or open thoracotomy. Indications for surgery are traumatic or spontaneous pneumothorax, hematothorax, cylothorax, failure of non operatif treatment, fibrothorax or schwarte limiting lung expansion, mesothelioma, and recurrent pneumothorax or malignant pleural effusion. Decision for surgery need appropriate timing and planning regarding different pathology and different etiology.

Keywords: pleural surgery, decortication, pleurectomy

1) Department of Surgery Faculty of Medicine Widya Mandala Catholic University Surabaya Indonesia, 2) Division of Thoracic, Cardiac \& Vascular-Endovascular PHC Teaching Hospital Surabaya Indonesia, 3) Department of Thoracic, Cardiac, \& Vascular Surgery Faculty of Medicine Universitas Airlangga Surabaya Indonesia

\section{INTRODUCTION}

Pleural disease is one of the most common diseases, every year in the UK there are 3000 people per million population affected. Pleural disease comes from a broad pathological scope and is a systematic approach and requires proper examination and management. ${ }^{3}$ Surgery in pleural disease has developed quite slowly, with many not following modern advances in pleural surgery. ${ }^{2}$ 
Techniques that are less invasive that can be used and provide good results are thoracoscopy and VATS (VideoAssisted Thoracic Surgery). VATS is very widely accepted among surgeons and is liked by many patients because the technique is less invasive. ${ }^{7}$

\section{Pleural Surgery in}

\section{Pneumothorax or Pleural Effusion}

Pleural effusion is a collection of fluid in the pleural cavity between the chest wall and the lung. This causes chest discomfort, coughing, or shortness of breath. ${ }^{24}$ Recurrent pleural effusion often occurs. This is caused by a process that is benign or malignant in the pleura. Management that can be done in the form of pleural drainage, thoracocentesis, pleurectomy, pleurodesis, or pleuroperitoneal shunt. ${ }^{24}$

Pleurodesis is a minor surgical procedure that can be a permanent solution this

A

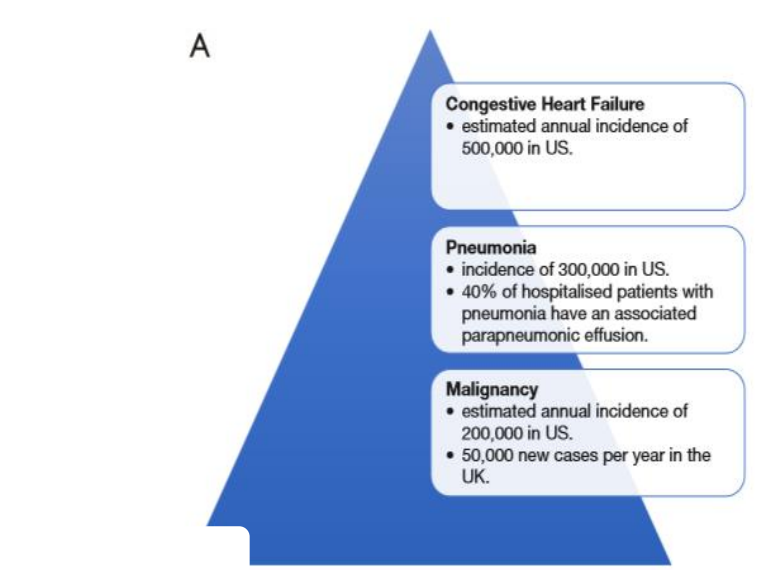

for reccurent malignant pleural effusion (MPE). This technique is done by inserting an inflammatory chemical into the pleural cavity which causes the formation of a scar between the lung and chest wall as an inflammatory response. This scar causes space in the lung and chest wall to be closed so that the fluid no longer collects here. The liquid inside can be absorbed more easily by the body. this technique has a success rate of $30 \% .^{23}$

\section{Pleural Surgery for Infection} (Empyema)

Empyema is an inflammatory process in the visceral or parietal pleura. Empyema has complex things to discuss and have unique clinical symptoms. there is a dominant inflammatory process, ptatological differences and there are various treatment techniques for

B

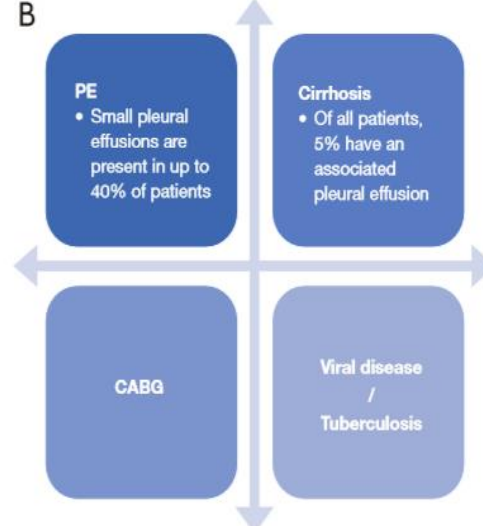

Figure 1 Causes ot pleural effusion. (A) Leading causes (2,3); (B) Common causes (4-6). PE, pulmonary embolism; CABG, coronary artery bypass graft. 
Figure 1. Cause of Pleural Effusion (Adopted from Perikleous P) ${ }^{14}$

Treatments for Recurrent Malignant Pleural Effusion
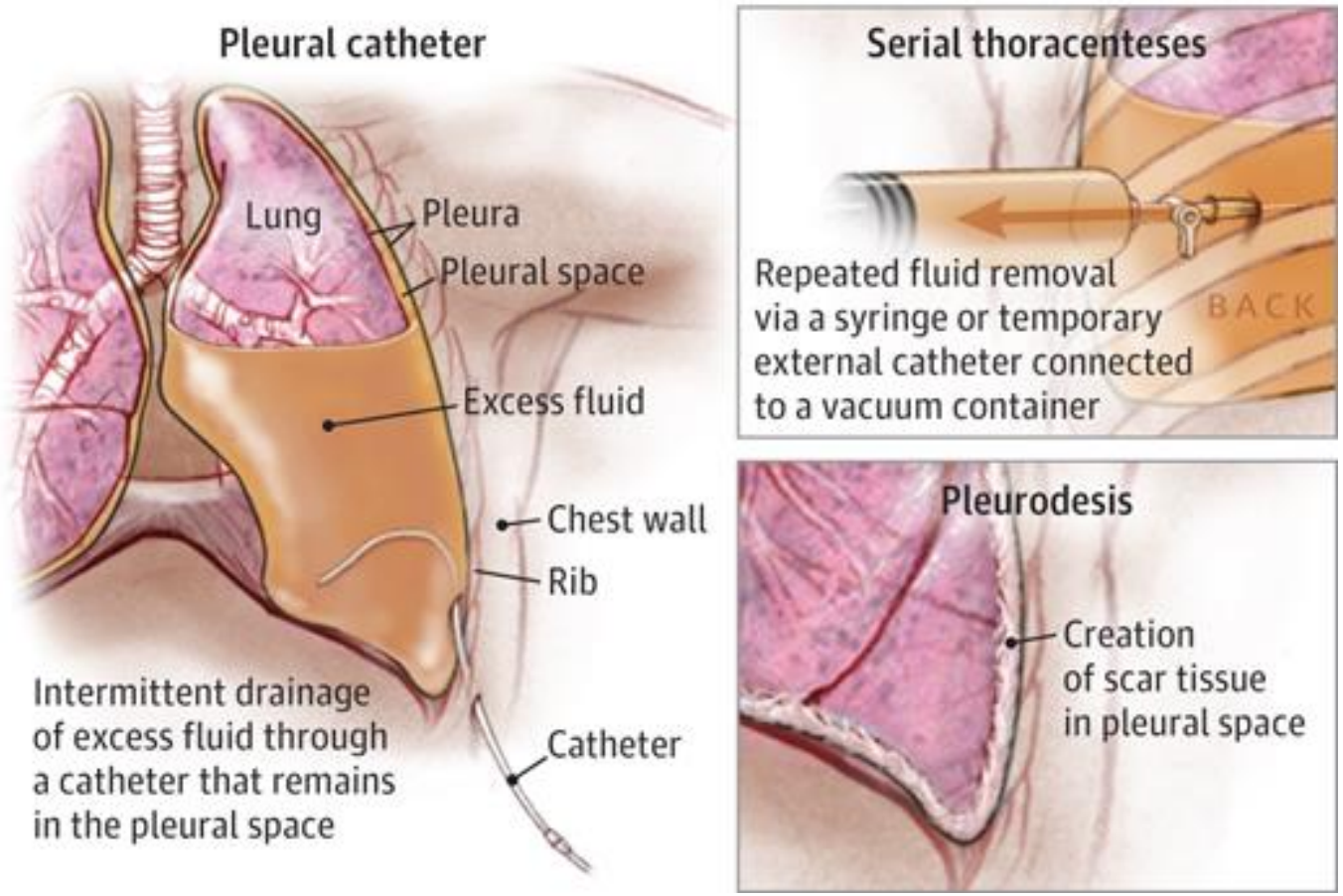

Figure 2. Treatments for Recurrent Malignant Pleural Effusion (Adopted From West H.

)$^{24}$ Pneumonia and subsequent parapneumonic effusion are the most common causes of empyema.

Other causes are penetrating or blunt chest injury, bronchogenic carcinoma, mediastinitis, esophageal rupture, expansion of below diaphragm, thoracic, and cervical spine infection, congenital cysts from the airway and infected esophagus. The use of broad spectrum antibiotics and vaccines for pneumococci should be given to patients with empyema. ${ }^{21}$

Patients with pleural empyema who have a risk of Methicilliin Resistent Staphylococcus Aureus and gram negative infection, can be given a third generation of cephalosporin (ceftriaxone) or aminopenicillin with $\beta$-lactamase inhibitors (ampicillin / sulbactam). Anaerobic organisms must be treated empirically, where ampicillin / sulbactam can be given to these organisms, whereas ceftriaxone must be given in conjunction with other drugs such as metronidazole. if anaerobic bacterial infection occurs in respiratory or upper gastrointestinal, clindamycin can be used instead of metronidazole. If postsurgical infection occurs, the antibiotics that can be used are vancomycin, flagyl and cefepime, or vancomycin and piperacillin / tazobactam (very adequate for Pseudomonas aeruginosa). For patients with a history or suspect extended spectrum $\beta$-lactamase-producing organisms can be 
given vancomycin and meropenem. Aminoglycosides are not recommended because they are not active in empyema fluid. Until now there is still no evidence base that proves that direct administration of antibiotics to pleural space provides better results than systemic antibiotics. ${ }^{21}$

Decortication is the treatment of choice for empyema with a lung that collapses and can't expand due to a thick sheath of inflammation. Decortication is usually used for tuberculotic management and post-traumatic trapped lung. This technique aims to restore lung elasticity and free pulmonary parenchyma from the pressure of the inflammatory sheath. In stage III empyema, there is a decrease in pulmonary perfusion of $20-25 \%$ on the infected side. Where stage III empyema is equivalent to empyema which has occurred more than 6 weeks. At this stage, FowlerDelorme's procedures were recommended, especially for postpneumonic empyema or accompanying trauma. ${ }^{12}$

Decortication can increase FEV1 from 50\% to $69 \%$ and vital capacity from $62 \%$ to $80 \%$. however, the function of the infected lung is still disrupted. Ventilation/lung perfusion improvement depends on multilevel functional lung damage. Patients with posttuberculotic with lung collapse and perfusion are minimal, decortication can be done, but the results are unpredictable. This technique is useful for patients with visible clinical symptoms and is not indicated for asymptomatic patients. ${ }^{12}$

\section{Pleural Surgery for Pleural Cancers}

Pleurectomy/decortication (P/D) are two sequences of procedures. Pleurectomy aims to open the chest and open the pleura around the affected tissue. While decortication to remove visible tumors from the lung surface and chest area. $^{6}$

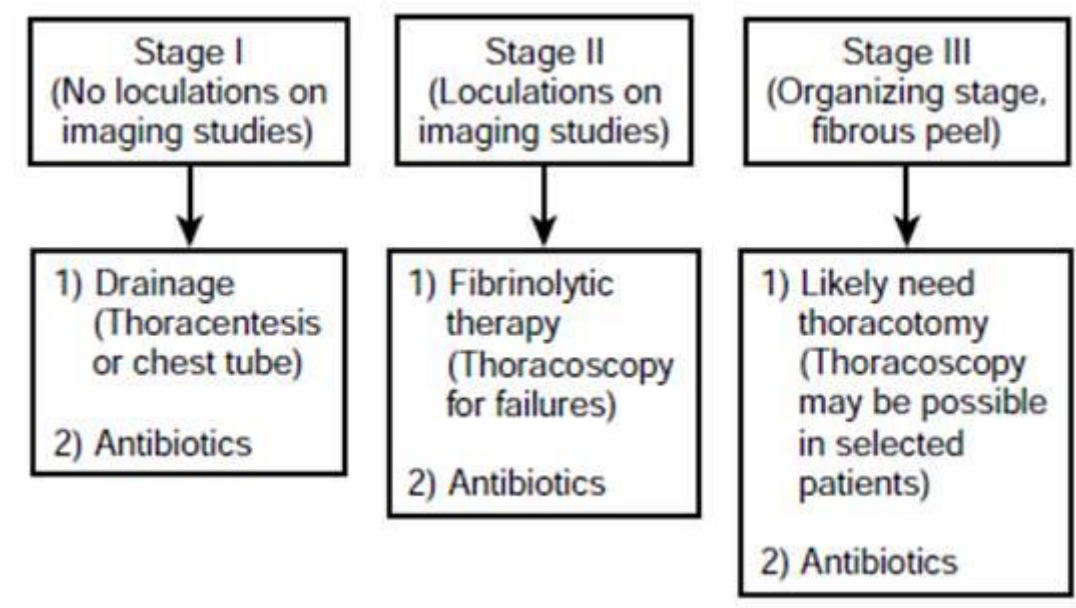

Figure 3. Stage of Empyema (Adopted From Mionar TF) ${ }^{12}$ 


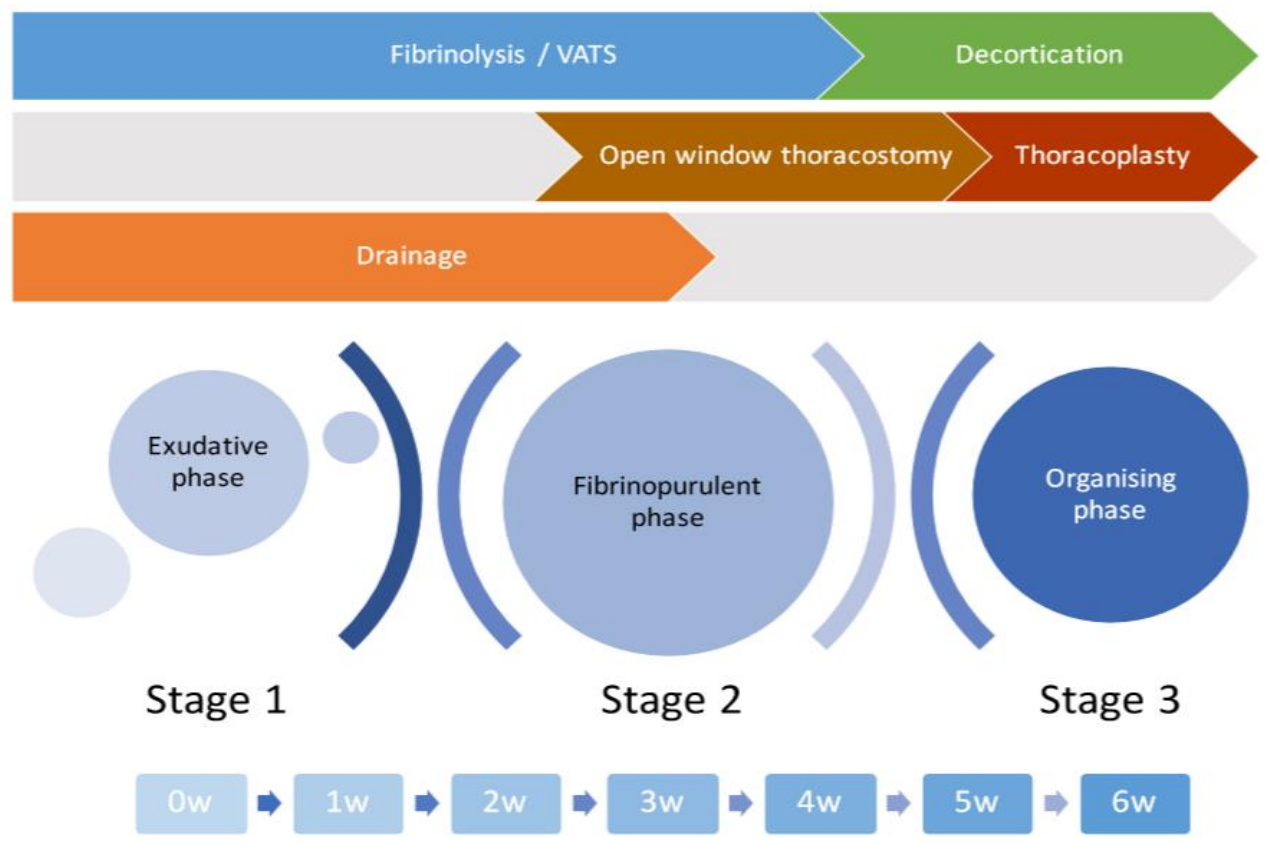

Figure 4. Time Scale for Different Stages of Thoracic Empyema and Treatment (Adopted From Perikleous P) ${ }^{14}$

Previously, pleurectomy was commonly used for the management of pleural mesothelioma to reduce fluid around the lungs and to reduce pain, but since the surgical technique developed, decortication can be done. Generally these two techniques are combined. This technique takes several hours to be completed and the success rate depends on the thoracic surgeon's skills. ${ }^{6}$

To be able to do major surgery in mesothelioma patients, the patient's condition must be stable and the mesothelioma he suffered is still in the initial stage. Pleurectomy/decortication can improve the survival rate and quality of life of patients, especially if combined with chemotherapy, radiation therapy, and other therapies. VATS (Video-assisted Thoracoscopic Surgery) is one of the treatment options and open surgery was performed if a larger tumor is found. $1,9,11,17,22$

\section{Pleural Surgery for Chylothorax}

Cylothorax is a collection of chylous fluid in the thorax cavity. This often occurs because of the leakage of lymphatic vessels in the thoracic duct. other causes are cardiothoracic surgery, penetrating or blund injury between the neck and upper abdomen, and other diseases such as lymphoma. The choice of therapy can be non operative and operative. $^{4,13,18}$ 


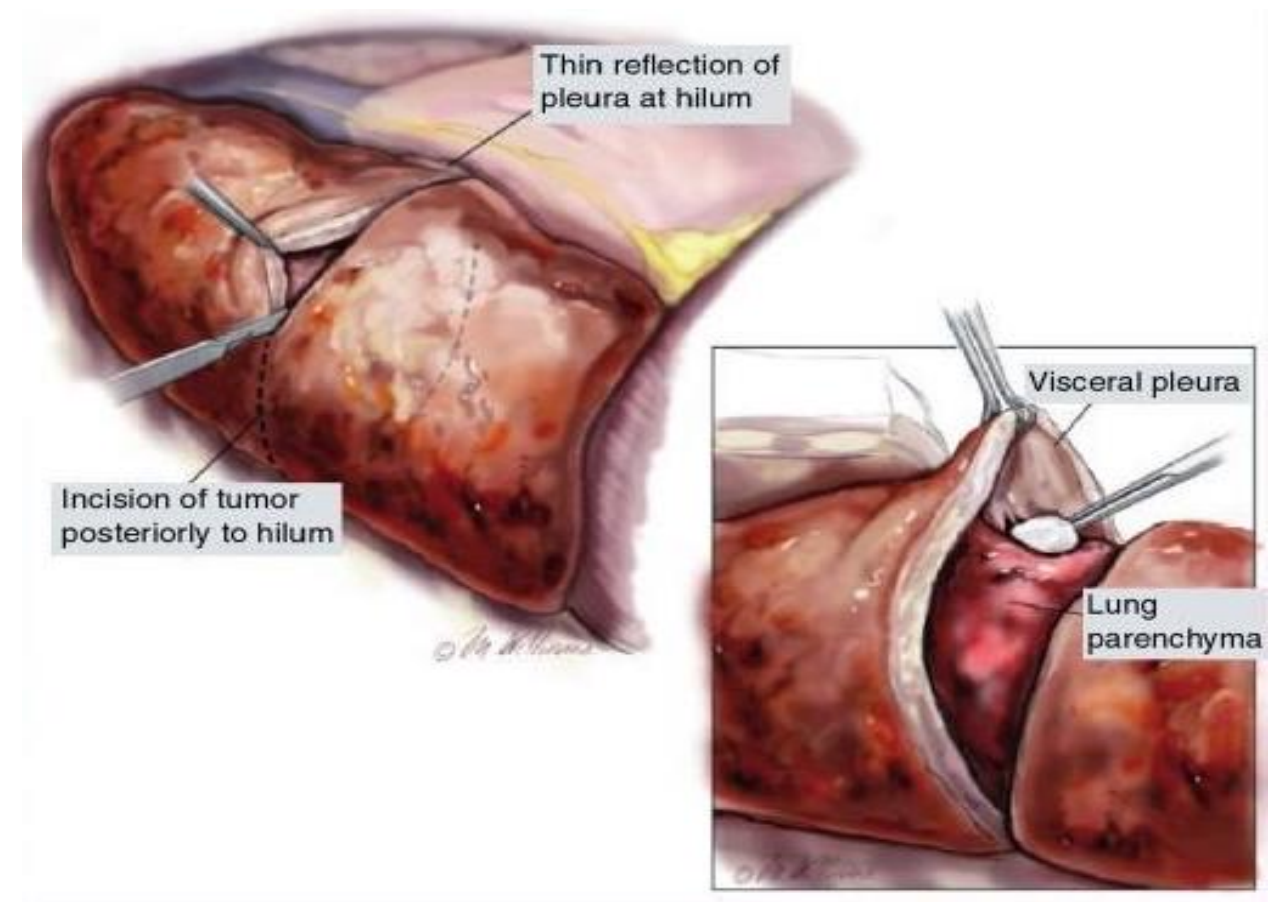

Figure 5. Pleural Surgery for Pleural Cancer (Adopted From Ismail-Khan R) ${ }^{6}$

The most preferred management is ligation of thoracic duct and it has a success rate of $95 \%$. Where this success rate also depends on the procedure performed either on the azygos vein or descending aorta and the structure around the posterior mediastinum. Thoracic duct is a thin structure, has a occasional peristaltic, and tubeshaped. This duct can be dissected or stapled or clipped. If there is sufficient length, this duct can be excised and histopathological examination is performed. Ligation of the supradiaphragmatic duct has a success rate of $90 \%$ but pleurodesis is not indicated in patients with trapped lung and prolonged effusion. ${ }^{10}$ there are several surgical options for thoracic duct ligation based on the location of the leakage and its etiology. Consuming milk or cream before surgery or injecting $1 \%$ evans blue dye on the thigh can clarify thoracic duct during VATS or open thoracotomy. ${ }^{16}$

\section{Pleural Surgery for Hematotoraks}

Hematothorax can occur due to trauma or non trauma. The most common causes are trauma, but non-traumatic causes such as aortic aneurysm, pulmonary embolism, haemostatic abnormalities or melignant metastatic disease can also occur. $^{5}$ VATS can identify and control bleeding and can clear the pleural cavity from the clots. In addition, chest tube was also needed. Open thoracotomy must be 

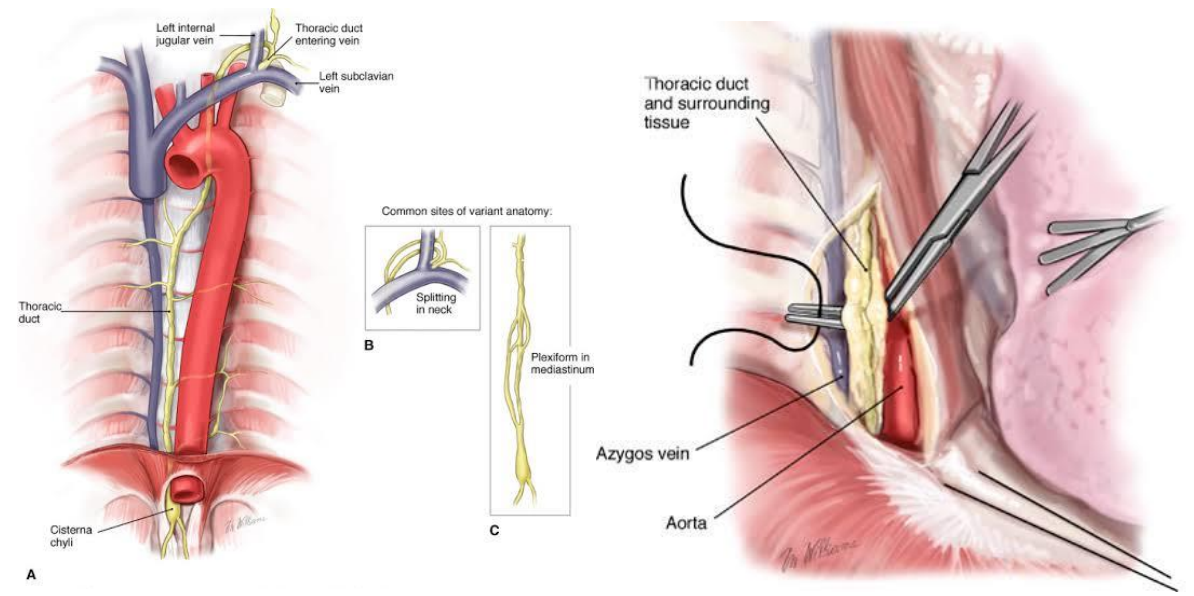

F igure 6. Surgery for Cylothorax (Adopted From Perikleous P) ${ }^{1}$

performed in patients with multiple trauma and massive bleeding. ${ }^{15}$ The clinical symptoms can be pain that is localized to an area that is inflamed or referred pain according to the nerve pathway Other symptoms can be difficulty breathing, sneezing, coughing, which exacerbates pain. Pain is described as sharp pain and gets worse with movement. ${ }^{8}$

Basically the parietal and visceral pleura have a smooth surface, but can be rough due to inflammation, so that at the physical examination friction rub or rough scratching sound can be heard during inspiration and expiration. ${ }^{8}$

There are several less invasive techniques for diagnosing and evaluating pelural disease, namely closed pleural biopsy, thoracosynthesis, guided image biopsy (CT or ultrasound), video-assisted Thoracoscopy surgery (VATS), medical thoracoscopy, and open thoracotomy.The main purpose of pleurisy therapy is (1) reducing pleuritic pain with analgesic drugs usually nonsteroidal anti-inflammatory drugs (NSAIDs) and (2) managing underlying diseases. ${ }^{8}$

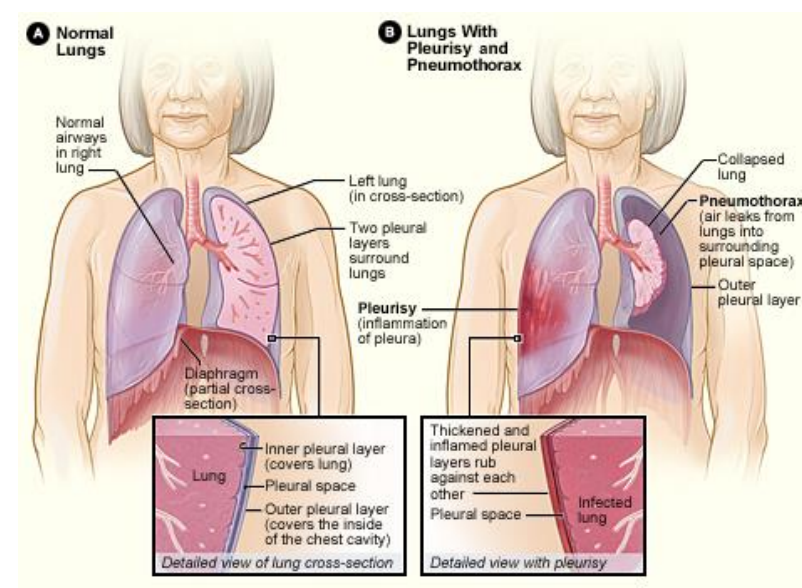

Figure 7. Lung With Pleurisy and Pneumothorax (Adopted From Kass SM) ${ }^{8}$ 


\section{CASE 1}

\section{Surabaya Pleural Surgery Experiences}
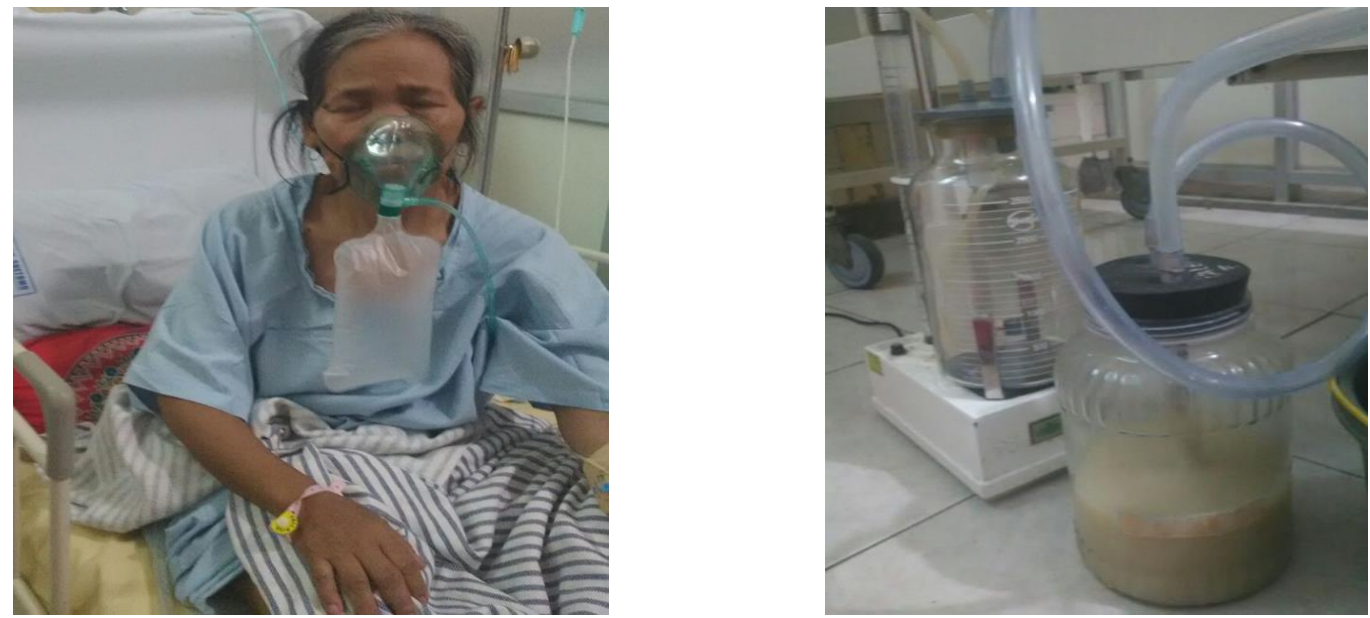

Figure 8. Female, 57 years old with Cylothorax. The patient initially had pneumonia and was treated in the lung room for 1 month. She also had right pleural effusion and drainage was perfomed. It appears cloudy white liquid like milk out of the drain as much as 1.5 liters. Pleural fluid analysis was done and the results of triglycerides 3 mmol / 1 (normal <1.24 $\mathrm{mmol} / \mathrm{l}$ ) and cholesterol 18 (normal $<5.18 \mathrm{mmol} / \mathrm{l}$ ).

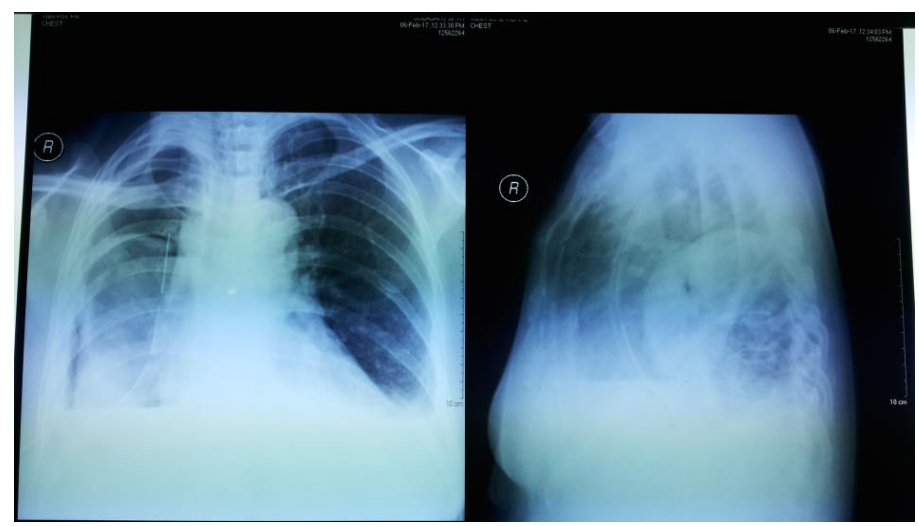

Figure 9. X-Ray Thorax, 6-2-2017

Table 1. Laboratorium Finding in Cylothorax

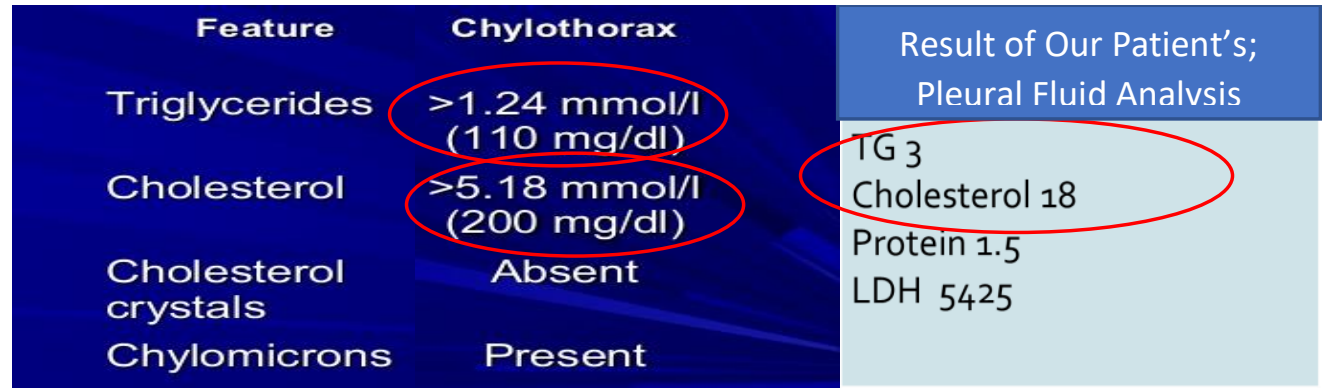




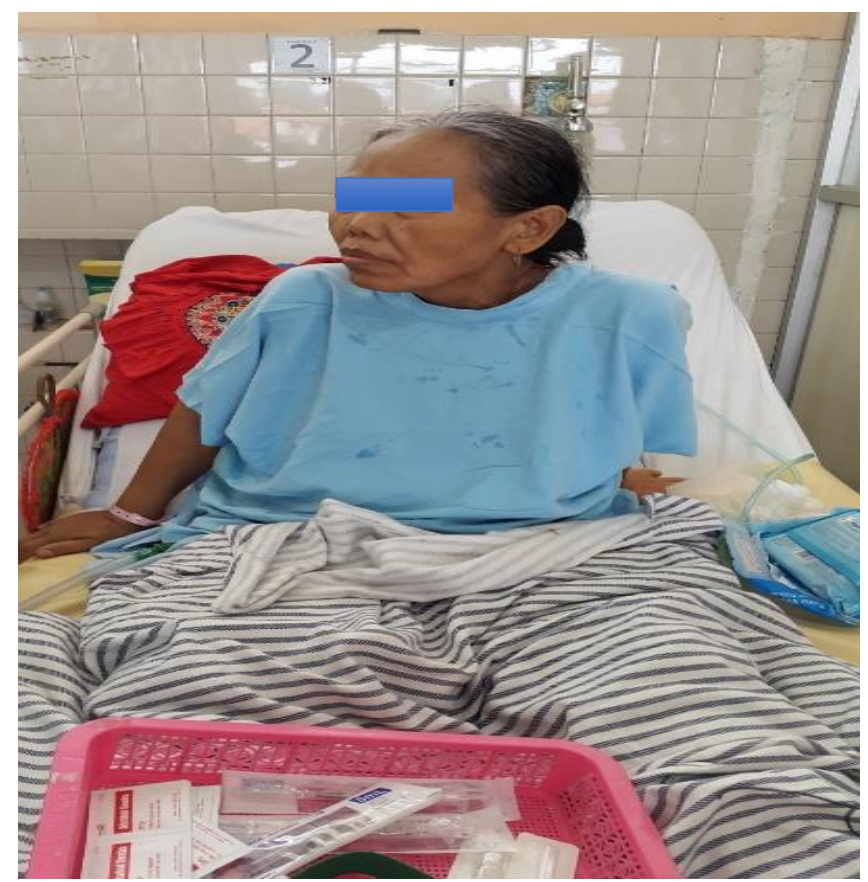

Figure 10. Patient condition after chest tube release

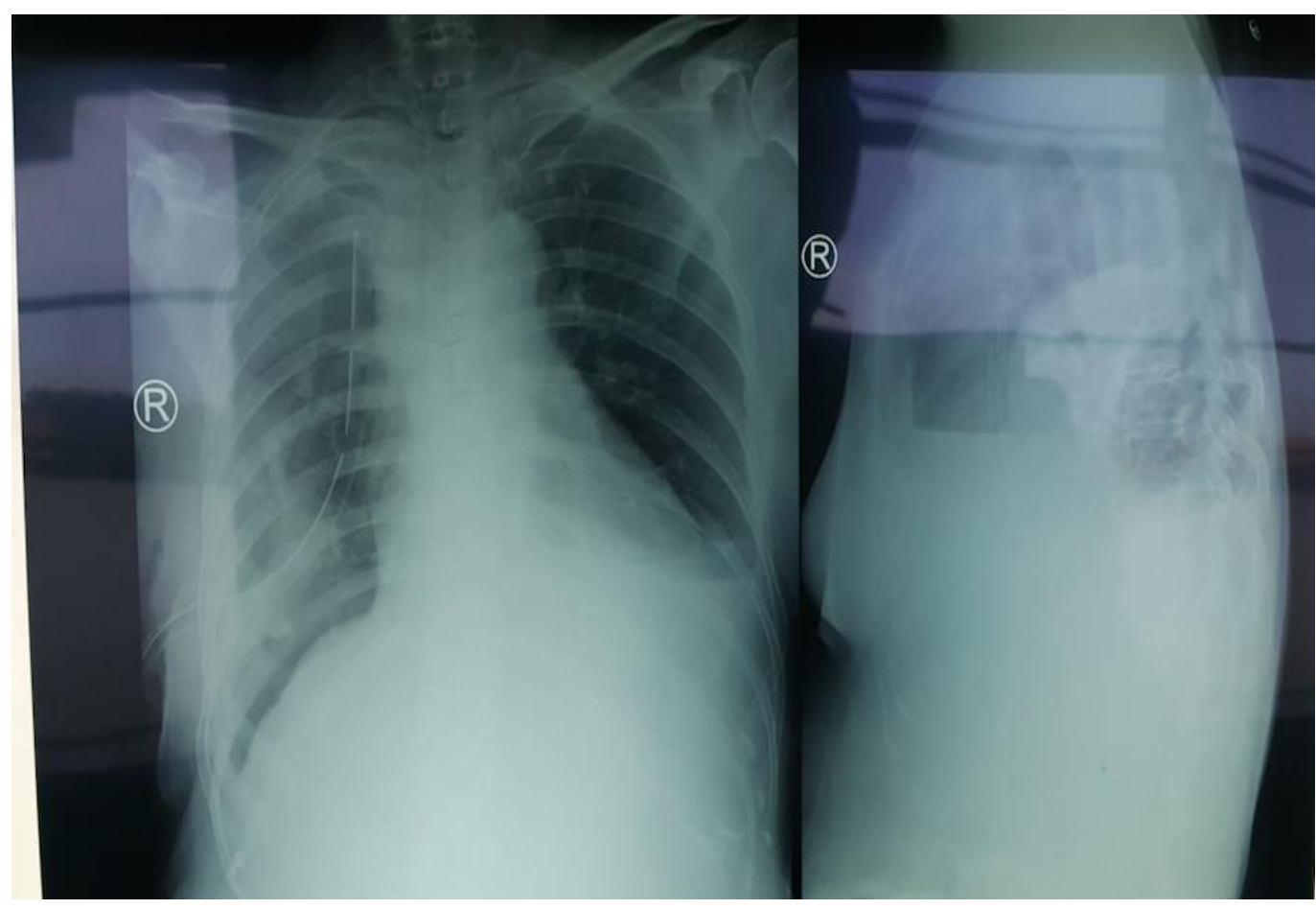

Figure 11. Chest X-Ray one month after release the chest tube 


\section{CASE 2}

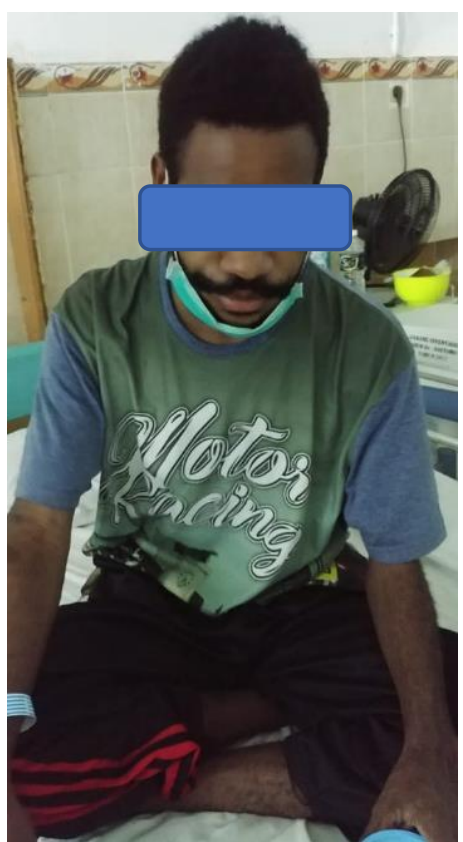

(a)

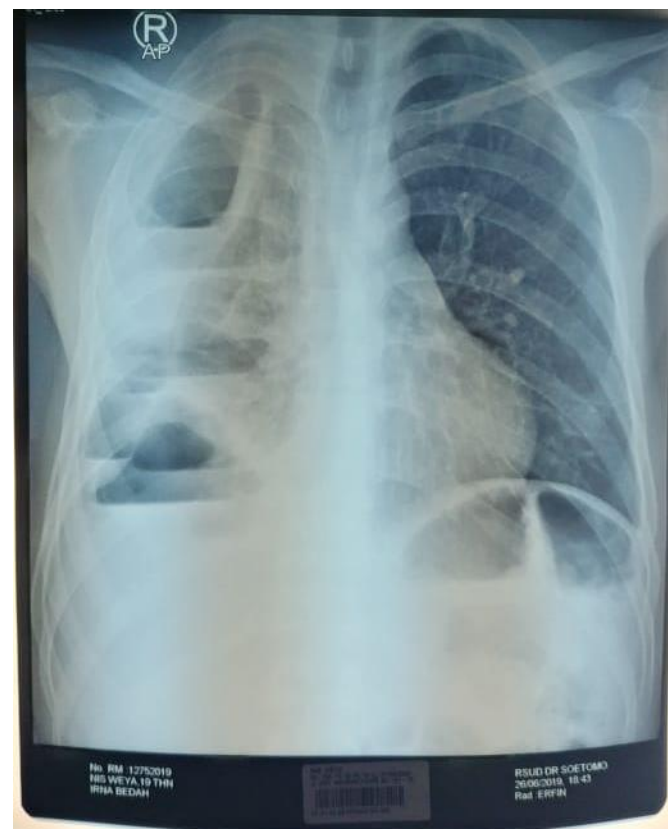

(b)

Figure 12. Man, 19 years old with Right Fluidopneumothorax + Pleuritis TB + Pulmonary TB with negative Acid Fast Bacilli (AFB) + Bronkiektasis pro Decortication, (a) Cilical Figure, (b) Thorax X-Ray with multiple air fluid level, thickening of the parietal and visceral pleura, and right lung collapse

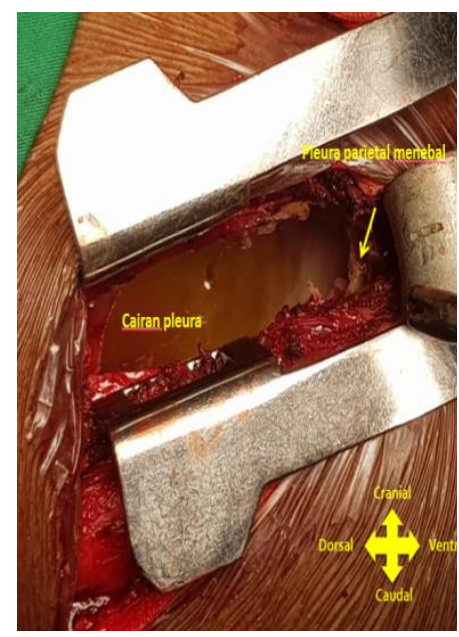

(a)

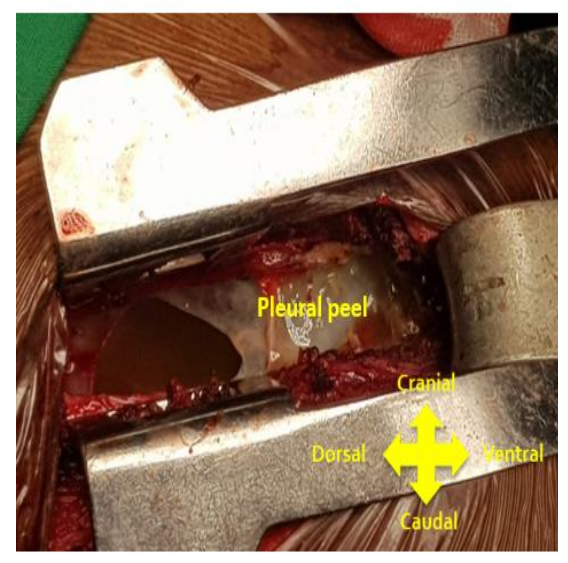

(b)

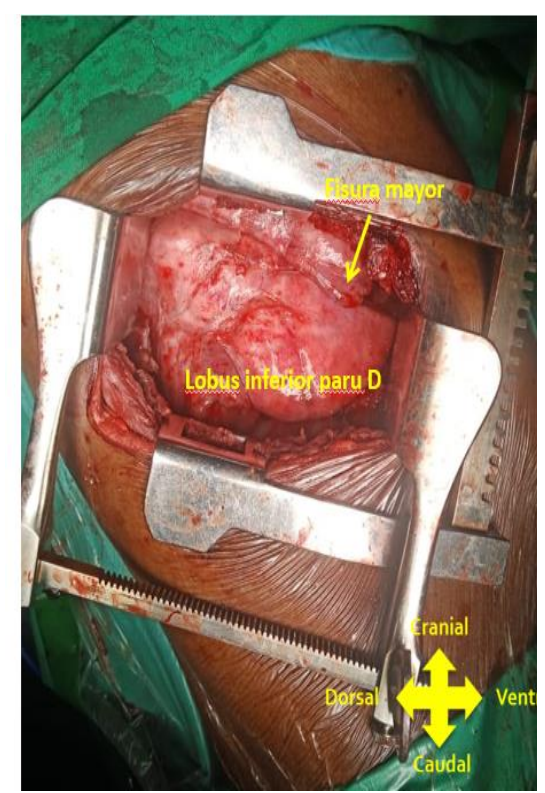

(c) 
Figure 13. Operating site (a) \& (b) before decortication seem fluid trapped inside the right thorax cavity, thickening of the parietal and visceral pleura, and right lung collapse, (c) after decortication the lung expand $\pm 80 \%$ and look reddish

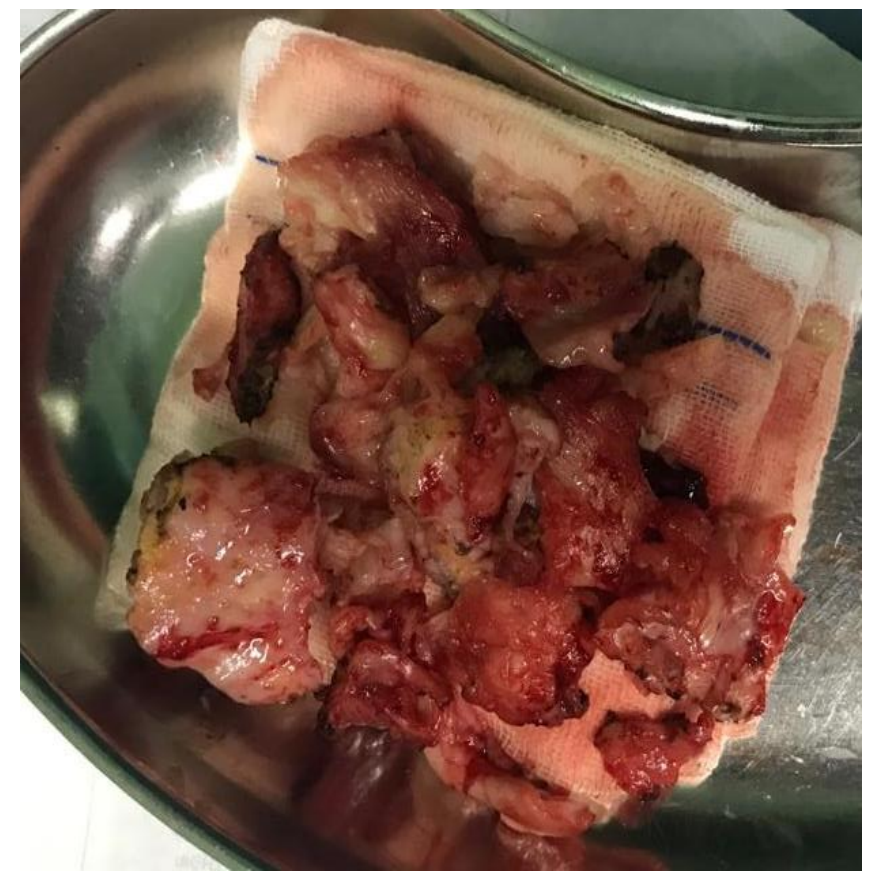

(a)

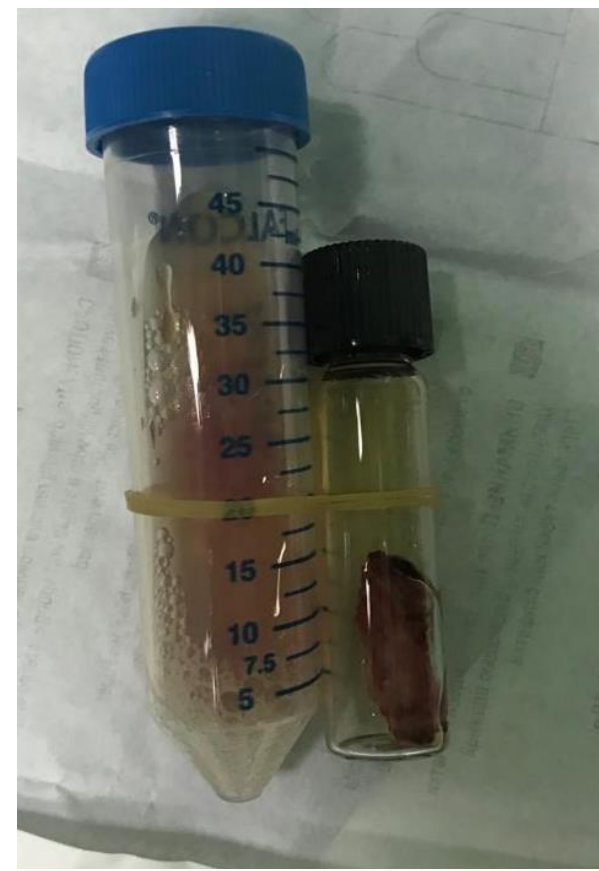

(b)

Figure 14. (a) Parietal and Visceral pleural peel (b) Pleural fluid for microbiology examination

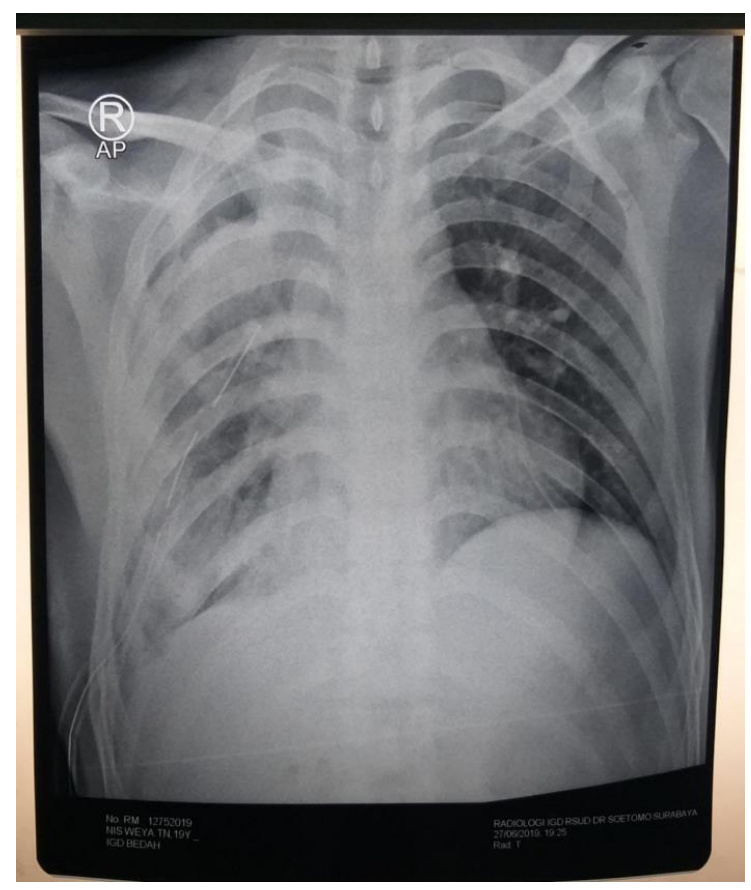

Figure 15. Chest X-Ray one day after Decortication, lung expand $\pm 80 \%$ 


\section{CASE 3}

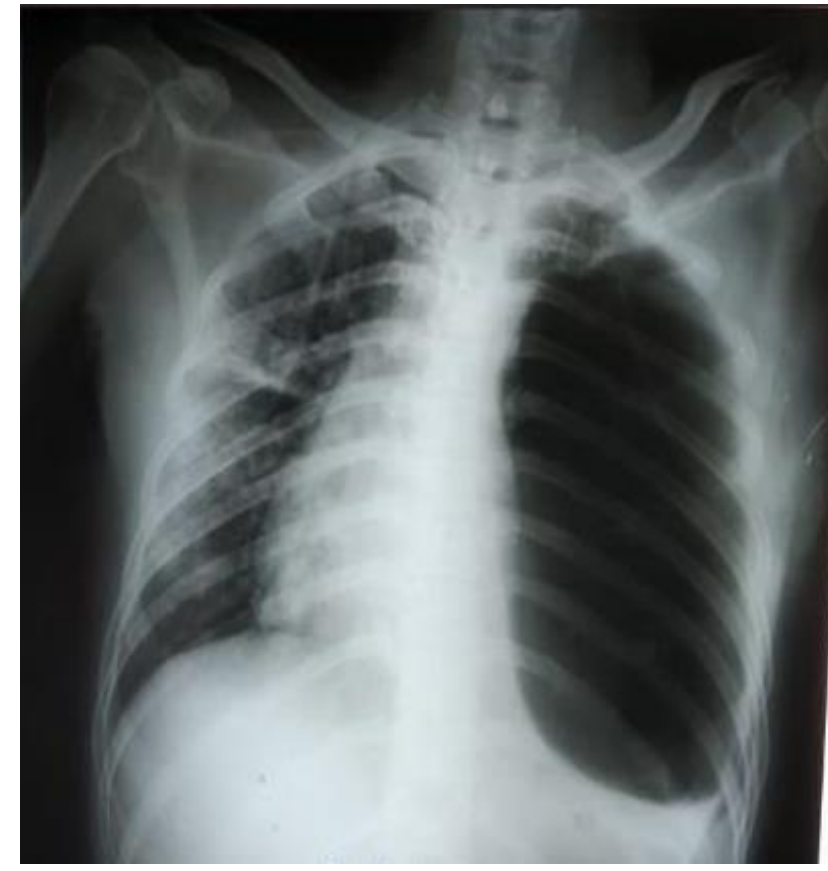

(a)

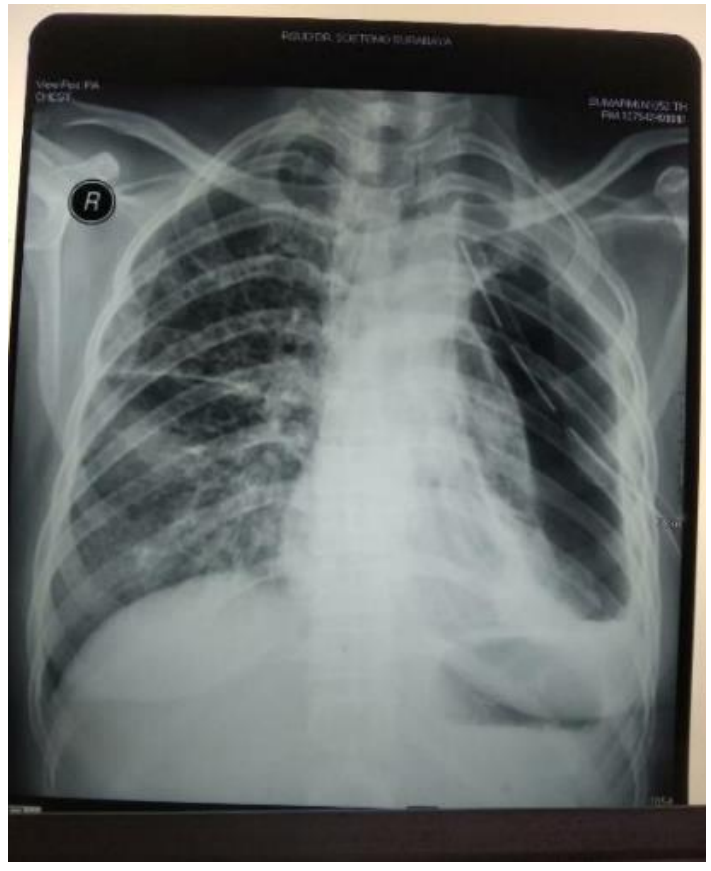

(b)

Figure 16. Woman, 50 years old with left fluidopneumothorax and Pulmonary TB, (a) before chest tube insertion seem thickening of parietal pleura, lung totally collapse, and left fluidopneumothorax (b) after chest tube insertion seem lung expand $\pm 30 \%$ and thickening of parietal and visceral pleura

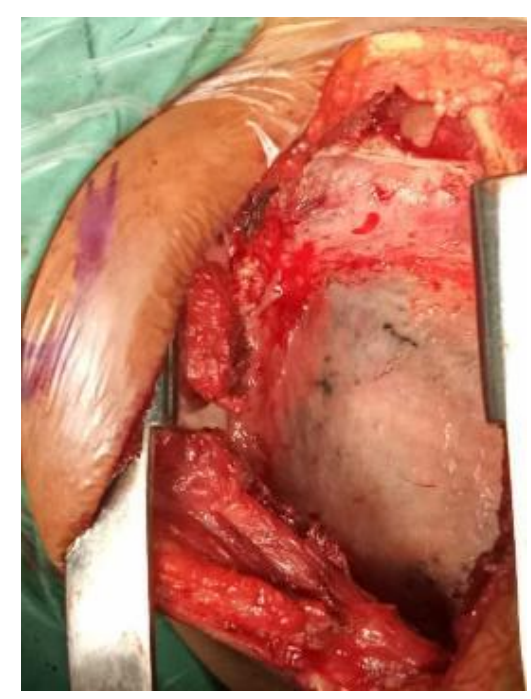

(a)

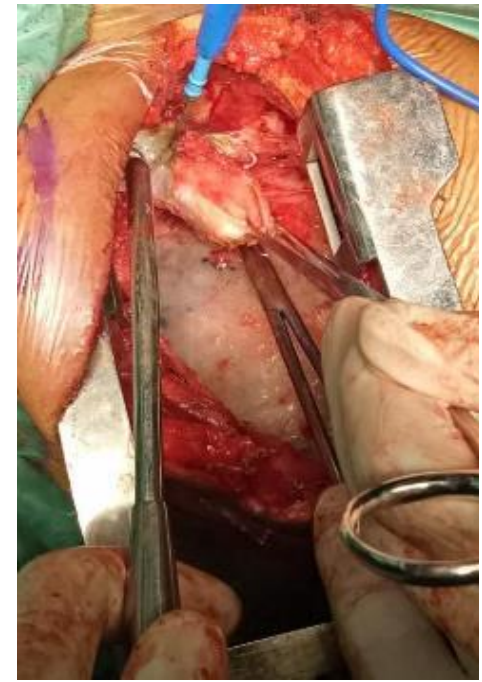

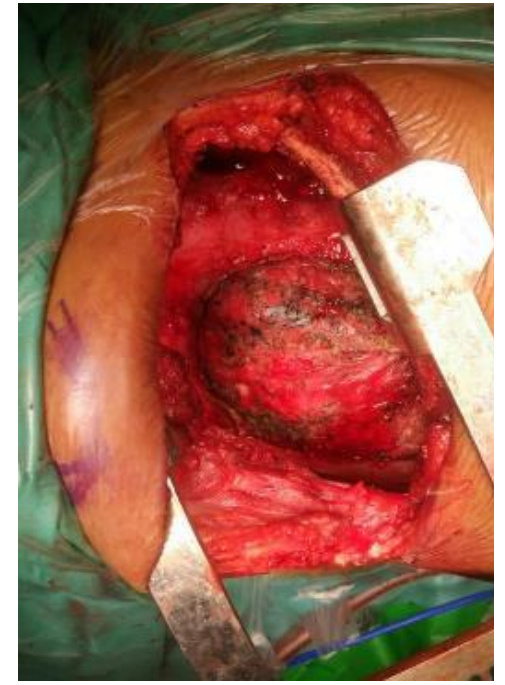

(b)

Figure 17. Operating site (a) before operation, lung collapse 30\%, (b) after operation lung expand $70-80 \%$ 


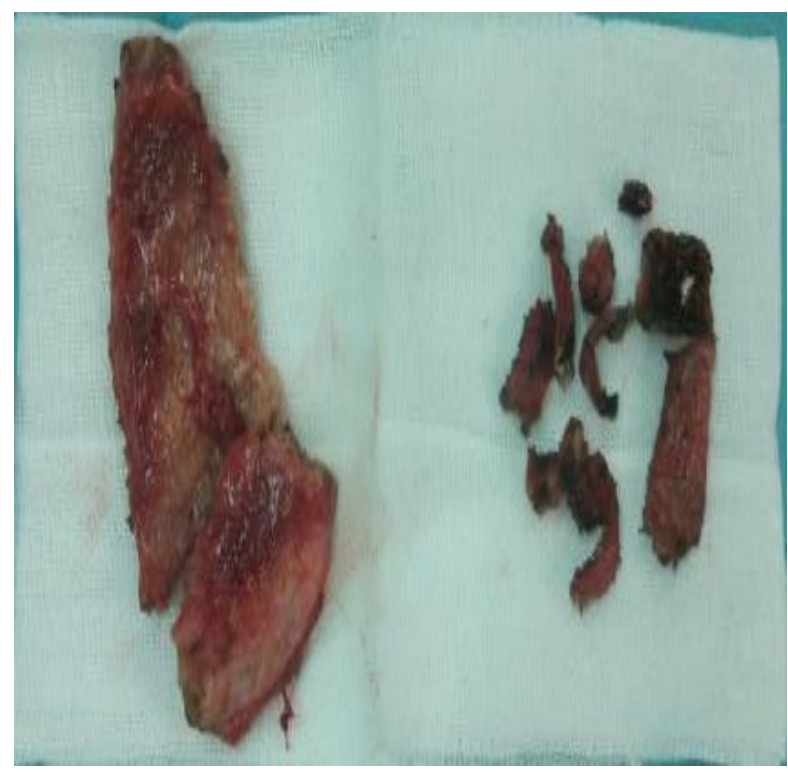

(a)

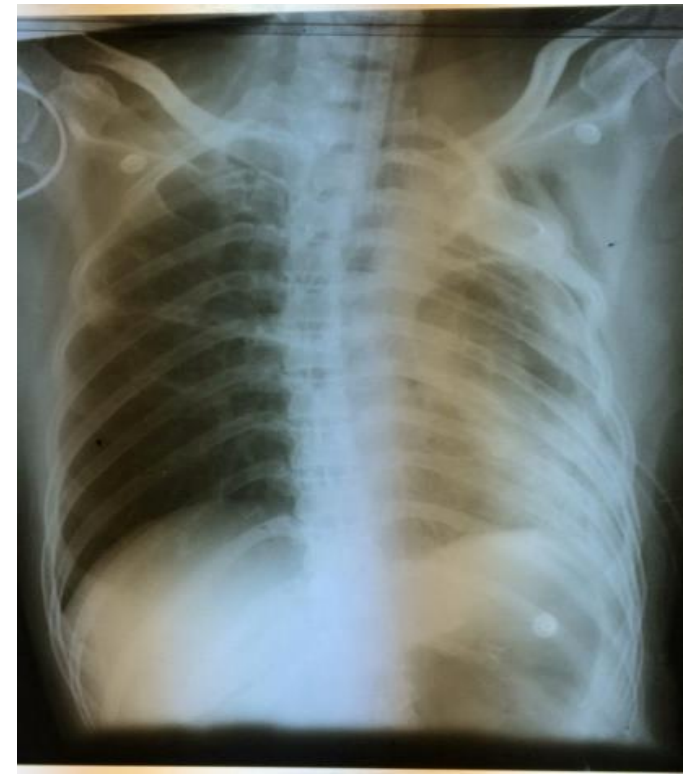

(b)

Figure 18. After decortication, (a) parietal and visceral pleura peel, (b) 1 week after decortication, chest tube was released and lung expand $80 \%$

\section{References}

1. Cardillo G, Carbone L, Carleo F, et al. Solitary Fibrous Tumors of the Pleura: An Analysis of 110 Patients Treated in a Single Institution. The Annals of Thoracic Surgery 2009;88:1632-7.

2. Davies MH. Recents Advances in The Surgery of the Lung And Pleura. British Journal of Surgery $1913 ; 1(2): 228-58$

3. Du Rand I, Maskell N. Introduction and methods: British Thoracic Society pleural disease guideline 2010. Thorax 2010;65(Suppl2):ii13

4. Fahimi H, Casselman FP, Mariani MA, et al. Current management of postoperative chylothorax. The Annals of Thoracic Surgery 2001;71:448-50.

5. Gallardo X, Castañer E, Mata JM. Benign pleural diseases. Eur $\mathbf{J}$ Radiol 2000;34:87-97.

6. Ismail-Khan $\mathrm{R}$, Robinson LA, Williams CC Jr, Garrett CR, Bepler G, Simon GR. Malignant pleural mesothelioma: a comprehensive review. Cancer Control. 2006 Oct. 13(4):255-63.

7. Johna S, Alkoraishi A, Taylor E, Derrick M, Bloch JH. VideoAssisted Thoracic Surgery: Applications and Outcome. JSLS : Journal of the Society of 
Laparoendoscopic Surgeons. 1997;1(1):41-44.

8. Kass SM, Williams PM, Reamy BV. Pleurisy. Am Fam Physician 2007;75:1357-64.

9. Kohler $\mathrm{M}$, Clarenbach $\mathrm{CF}$, Kestenholz P, et al. Diagnosis, treatment and long-term outcome of solitary fibrous tumours of the pleura. European Journal of CardioThoracic Surgery 2007;32:403-8.

10. Lee KH, Jung JS, Cho SB, et al. Thoracic Duct Embolization with Lipiodol for Chylothorax due to Thoracic Endovascular Aortic Repair with Debranching Procedure. The Korean Journal of Thoracic and Cardiovascular Surgery 2015;48:74-7.

11. Liu J, Cai C, Wang D, et al. Videoassisted thoracoscopic surgery (VATS) for patients with solitary fibrous tumors of the pleura. $\mathbf{J}$ Thorac Oncol 2010;5:240-3.

12. Mionar TF. Current surgical treatment of thoracic empyema in adults. European Journal of CardioThoracic Surgery 2007; 32(3): 422430

13. O'Callaghan AM, Mead GM. Chylothorax in lymphoma: mechanisms and management. Ann Oncol 1995;6:603-7.

14. Perikleous P, Rathinam S, Waller D.A. VATS and Open Chest Surgery in Diagnosis and Treatment of Benign Pleural Diseases. Journal of Visualized Surgery 2017;3:6-10.

15. Richardson JD, Miller FB, Carrillo $\mathrm{EH}$, et al. Complex thoracic injuries. Surg Clin North Am 1996;76:725-48

16. Robins E, Walsh D. Minimally Invasive Surgical Treatment of Pediatric Chylothorax. Society of American Gastrointestinal and Endoscopic Surgeons 2017: 5.

17. Robinson LA. Solitary fibrous tumor of the pleura. Cancer Control 2006;13:264-9.

18. Romero S. Nontraumatic chylothorax. Current Opinion in Pulmonary Medicine 2000;6:28791.

19. Schild HH, Strassburg CP, Welz A, et al. Treatment options in patients with chylothorax. Dtsch Arztebl Int 2013;110:819-26

20. Sensakovic WF, Armato SG, Starkey A, et al. Quantitative measurement of lung reexpansion in malignant pleural mesothelioma patients undergoing pleurectomy/ 
decortication. Acad Radiol 2011;18:294-8.

21. Shen RK, Bribriesco A, Crabtree T, et al. Guidelines for The Management of eEmpyema. The American Association fot Thoracic Surgery Consensus 2017: 5-6

22. Takahama M, Kushibe K, Kawaguchi T, et al. VideoAssisted Thoracoscopic Surgery Is a Promising Treatment for Solitary
Fibrous Tumor of the Pleura. Chest 2004;125:1144-7.

23. Vaz MC, Marchi E, Vargas FS. Pleurodesis: technique and indications. $\quad \mathrm{J} \quad$ Bras Pneumol.2006;32, (4): 347-356

24. West H. Malignant Pleural Effusions. JAMA Oncol. 2015;1(2):260. 